\title{
A Traffic Simulation Tool for Assessing Smart City Policies (CitScale)
}

\author{
M. P. Linares ${ }^{1}$, L. Montero ${ }^{1}$, J. Casanovas ${ }^{1,2}$ \\ ${ }^{1}$ Universitat Politècnica de Catalunya - BarcelonaTECH \\ 2 Barcelona Supercomputing Center (BSC-CNS) \\ Barcelona, Spain \\ mari.paz.linares@upc.edu, lidia.montero@upc.edu, josep.casanovas@upc.edu
}

Over the last century, cities have developed as a function of increased usage of automobiles as the standard transport mode. The number of cars increased along with the population as highways and parking spots became essential in city planning. Now, there is more focus on how the existing infrastructure could be used as efficiently as possible rather than increasing capacity by merely building new roads. An important part of traffic planning is a sustainable transport system, which thereby reduces congestion and emissions by using the available capacity in a more efficient way.

Traffic simulation models are tools for assessing new mobility solutions and analysing changes in the infrastructure, such as rearranging intersections and building new roads. Transportation is undergoing a profound and significant transformation as it seeks to fulfil the promise of connected mobility for people and goods while limiting its carbon footprint. Physical changes to the road network mean large investments that must be comprehensively considered before acting. Modelling different scenarios of infrastructural changes allows making forecasts without any physical changes. Autonomous vehicles are potentially changing the economics of ownership as well as the use of the transportation networks, which will likely accelerate trends towards greater use of app-based ride hailing and/or sharing by private transportation network companies. American and European cities are seeing a rise in several potential business models with varying degrees of ride sharing and public vs. private involvement in delivering mobility services (MaaS). Implications for transit agencies and mobility service providers must be evaluated, and this can be done by traffic simulation models that provide a model-based framework for evaluating the mobility impact of new services.

Today, data is generated on a very large scale by a wide range of sources such as sensors, embedded devices, social media, and audio/video. Advances in storage technologies and their continuously falling prices allow collecting and storing huge amounts of data for long periods of time, creating entirely new markets for evaluating this data. Recent studies compare the value of data to modern business as equivalent to the value of oil, even going so far as to refer to data as "the new oil" 1 . However, as is the case for crude oil, data in its raw form is not very useful. To transform crude oil into value, a long evaluation chain composed of heterogeneous transformation steps needs to be applied before oil is converted into the essential energy source we all so heavily rely on. The process of turning raw data into valuable insights is referred to as data analytics. Big data have been a significant new resource in many fields over the last decade, and most current analytic techniques process the data through a pipeline: they first extract the data to be analysed from different sources, e.g., databases, social media, or stream emitters; they copy them into some form of usually immutable data structures; a stepwise process is applied; and then output files are produced or social media is minded for trends (even in near real-time). While this is very suitable for tasks like sorting vast amounts of data, huge logs analytics is less suitable for domains such as urban transportation with its complicated relationships between different agents characterizing urban mobility. Simulation model-driven approaches are powerful because they rely on a profound understanding of the systems and processes, thus providing benefits from scientifically established relationships.

According to reputed studies [1], Berlin and London have similarly shifted away from traditional patterns of urban mobility. They are dynamic cities undergoing extensive socio-economic pressures with high levels of national and international immigration and related processes of inner-city gentrification. Both cities have forward-thinking city governments that have implemented progressive land-use and transport planning policies through investing heavily in public transport, walking, cycling and the public

\footnotetext{
${ }^{1}$ P. Rotella. "Is data the new oil?"( http://www.forbes.com/sites/ perryrotella/2012/04/02/is-data-the-new-oil/)
} 
realm. Furthermore, both cities have thriving tech industries and are using this economic specialization to foster innovation in electric vehicles, car sharing schemes, smart cards and mobile travel apps. The combination of these shared factors means London and Berlin can be considered "ahead of the curve" in terms of changing travel patterns. The pace of digitalisation in transport, especially in cities, has accelerated in recent years as many new technologies have been introduced and citizens have adopted new behaviours. Both within and outside the transport sector, technology has become conflated with evolving societal trends and new relationships centred around the production and consumption of services, which has occurred more quickly than many authorities anticipated, thus outpacing the speed of regulatory adjustments. These are real challenges for public authorities, and it is likely that the present disruptions foreshadow even greater ones in the future.

Metropolitan travellers usually have a variety of transport modes to move from their origin to their destinations. When deciding which transport modes to use, they consider a number of criteria such as: cost; travel time; flexibility to changes in trip planning; and convenience (distances from origins to the starting point of the journey or from the end of the journey to the destination); among others. The shared journey (ridesharing, trip sharing) modality refers to a mode of transport in which single travellers share a vehicle and the associated costs for making a journey. From a conceptual point of view, it is a system that can combine the flexibility and speed of private vehicles with the low cost of the fixed public transport system while also favouring convenience. Many of the existing services have emerged in an informal and spontaneous way, more as a result of ingenious private initiative than as a consequence of conducting a study and developing a subsequently appropriate design. As a result, the coordination of vehicle sharing services is a casual and disorganized activity that works best when used as a regular transport alternative. The greatest challenge still lies in coordinating and timing itineraries in a systematic way that explicitly takes into account user requirements and interests [2]. A practical example of rethinking public transport via the concept of transport on demand [3] is given by the KUTSUPLUS project in Helsinki.

This has become a "hot trending topic", not only because it represents a potentially very efficient way of restructuring mobility services, but because from the perspective of sustainable urban mobility it can represent a very efficient way to reduce the number of private vehicles on a road network. The reason for this is that shared vehicle services can reduce the use of private vehicles and thus reduce congestion, environmental impacts and energy consumption while increasing service efficiency. The International Transport Forum (ITF) of the OECD [4]-[6] has declared this to be one of the key issues in future mobility. The papers by Ma et al. [7] and Boesch et al. [8] illustrate these research efforts.

A glimpse at the potential of new mobility services has been provided by transportation data analytics based on ICT technologies as well as by reports from Lisbon, Helsinki and Auckland [4]-[6]. These relied on the simulation of working day scenarios, including the current modes and the different adoption rates of the new shared services, all of which were examined to assess how the optimized use of new shared modes can change the future of metropolitan mobility. Traffic simulation provides a detailed array of indicators that allow measuring impacts on the city and on the transportation system, such as: decreases in CO2 emissions; required parking spaces; car use; congestion; changes to accessibility; the extent of the modal shift; and the performance of new shared services, both from a user perspective (travel times, waiting times, access times, number of transfers) and on the operator/production side (number of vehicles, occupancy, depot location and sizes, costs). The indicators produced for different scenarios increase our understanding of the policy implications emerging from these new services. The ultimate goal of those documents is to provide local authorities and other public officials with meaningful advice for the challenges and opportunities brought by these new services.

\section{Why CitScale?}

Simulation is a technique [9] in which a sampling experiment is performed on a dynamic real system formally represented on a computer model. Simulation assumes that the model evolves over time in a proper imitation of the modelled system's evolution. Thus, samples of the observational variables of interest are collected, which are used then to draw conclusions on the system behaviour by means of statistical analysis techniques. From a methodological point of view, it is necessary to analyse several replications of each scenario, with the set of scenarios being defined through an experimental design that considers different policies and management of MaaS services.

A huge amount of data is involved in analysing the traffic simulation results delivered by microscopic traffic simulation models. Traffic simulation platforms are currently based on shallow graphical outputs for analysing their results; however, they are limited in terms of customization for comparing and jointly describing the basic Key Performance Indicators (KPI) for a set of replications in an experimental design. 


\section{CitScale: a visualization tool based on traffic simulation}

The CitScale tool is a simulation-based platform developed in inLab FIB at Universitat Politècnica de Catalunya (UPC) [10] for visualizing, analysing and comparing experimental designs that simulate urban scenarios of new mobility concepts and new automotive vehicles. This platform provides a graphical interface to compare and display traffic simulation results.

CitScale [11] is interactive and performs analytics on traffic simulation results after completing the executions. In addition, it can be accessed from any browser without any need of a traffic simulator on the user side. It allows simultaneous access to the data results from different experiments and simulation models. The system assists analysts in evaluating and comparing potential scenarios that apply new mobility vehicle/concepts and policies. CitScale is based mainly on the development of geo-located map visualizations in order to be able to improve the interpretation of simulation results, which helps the analysts who work daily on these types of projects; but it also supports demonstrations for clients and visitors. A prototype service for calculating static and time-dependent shortest paths has also been developed and integrated.

\section{CitScale: A Simulation Testbed}

Figure 1 shows the proposed simulation testbed, which is composed of an Executions Controller, a Traffic Simulation module, a Result Processing module and a Visual Analytics module. The visualization and analysis tool has been implemented using Shiny [12], a web application framework for $\mathrm{R}$ that simplifies the development of simulation results analysis into interactive web applications. The shiny web application is agnostic to the traffic simulation platform. In order to emulate new vehicles and concepts, several components in the traffic simulation model must be developed using API extensions. The simulation results analysis is performed by two fundamental and independent components: a Result Processing module and a Visual Analytics module. Both are essential for properly automated functioning: one handles the automated preparation of data and the other the data visualization. In addition, the Result Processing module in Figure 1 covers the entire pre-processing of data automatically. In this way, the Visual Analytics module can use the results of the traffic simulation environment directly without need for a manual update.

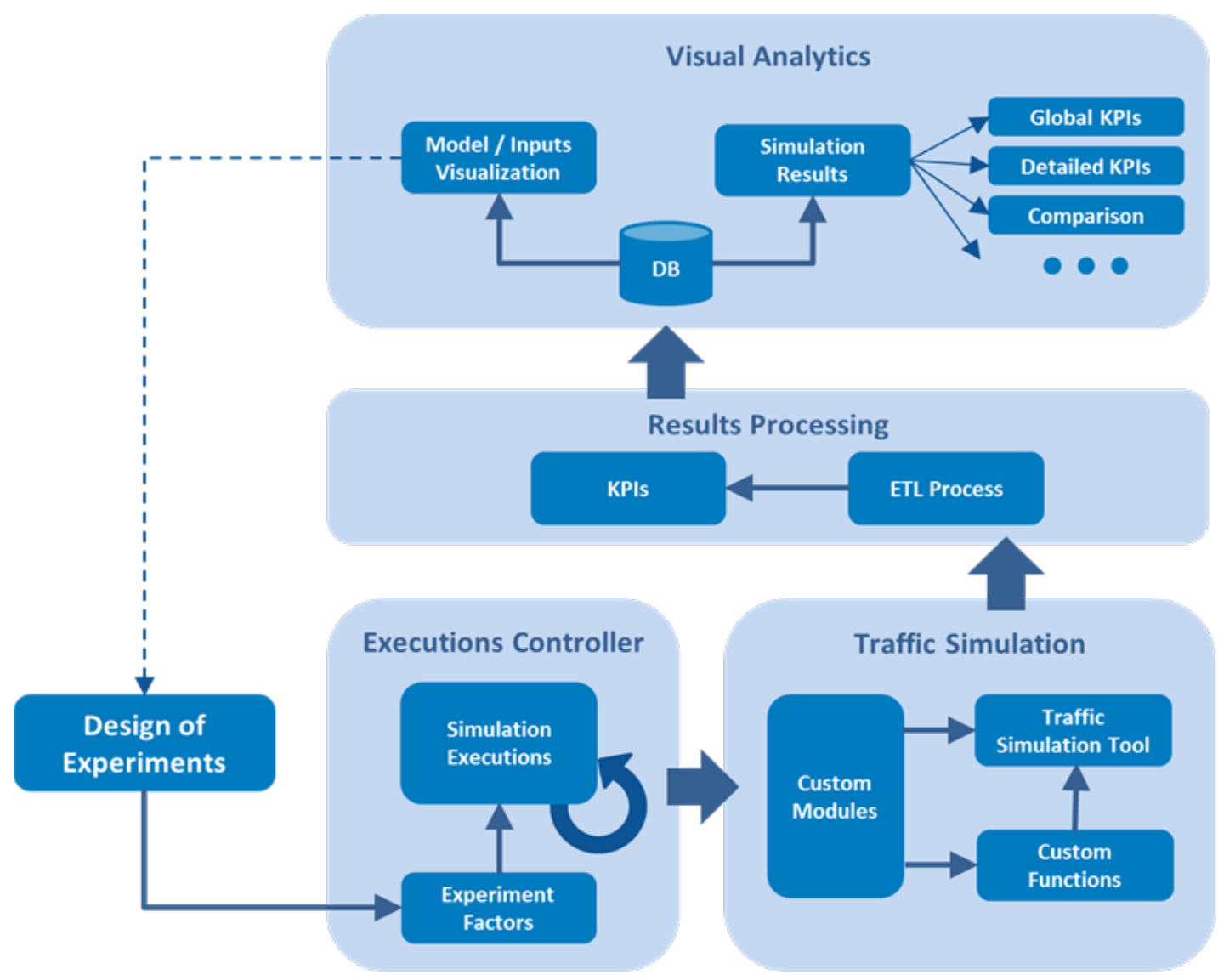

Fig. 1. Simulation Testbed Architecture. 


\section{Traffic Simulation Environment}

The Traffic Simulation Environment of the framework (Figure 2) is composed of a traffic simulation tool and a set of auxiliary modules and functions that customize the traffic model. This serves to extend the standard functionalities of the simulation tool, which allows working with the specific requirements of the different analysed case studies.

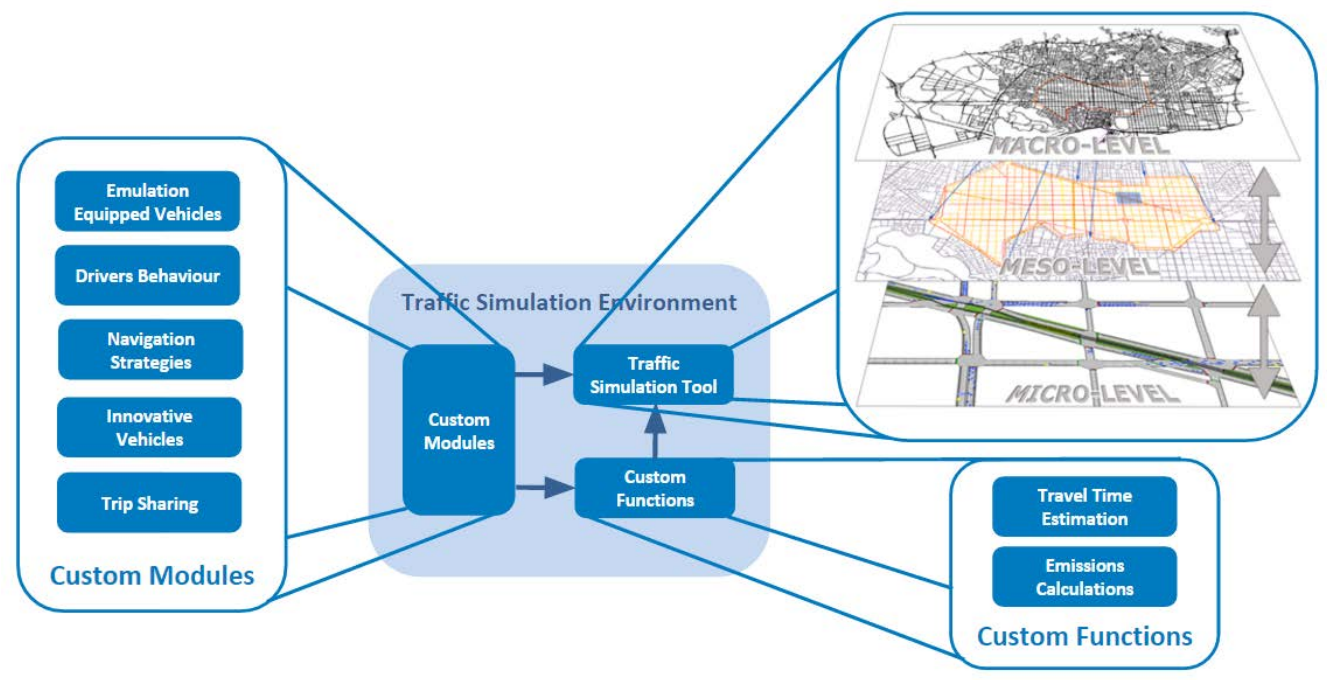

Fig. 1. Traffic Simulation Environment.

Traffic and transportation modelling applies the same steps as any generic methodology for model building [13]. Mobility must be understood as a social and economic phenomenon, a consequence of human activities distributed across space and time. These activities generate the need to move people and freight between various points, which in turn generate the trips to accomplish them. The demand is the first main component in any transportation system model, and it can be considered in various ways. CitScale supports an aggregated representation of the demand, based on origin-destination matrices between transportation analysis zones for a time period and user class. User class can refer generically to either a purpose, to a vehicle type, or to a combination of both.

Another main component of transportation models is the supply network and its conditions of use, specifically in terms of traffic management and control policies. The network is modelled in a way that depends on the objectives of the transportation system analysis. CitScale supports either microsimulation network models (very detailed representation), or mesoscopic network models consisting of:

- Different degrees of detail in the network geometry, ranging from a simple graph representation to a very detailed representation of the geometry, depending on the modelling approach and the model's purposes.

- An explicit description of the traffic control settings.

- Possibly other objects (detectors, variable message panels, etc.), depending on the model's objectives.

The pervasive penetration of new vehicles and concepts must be evaluated by traffic models capable of emulating:

- Replacement of fossil fuel propelled vehicles with vehicles that have more efficient engines propelled by alternative or green energy sources.

- Similar scenarios that have been drawn up for connected vehicles when the main goal is to replace old vehicles with new ones. The main challenge in these cases is estimating the impacts when a combination of conventional, connected and/or autonomous vehicles coexists.

- Urban policies that promote citizens riding bicycles and making the city safer for them as an important component of sustainability action plans. In order to shift from private cars to shared bicycle systems and lightweight three-wheeled electric scooters, among others, it is generally necessary to establish a segregated bicycle network throughout the city. 
- New mobility concepts such as those under the Mobility-as-a-Service (MaaS) paradigm, car-sharing and multiple passenger trip-sharing. These indicate a shift away from personally owned modes of transportation and towards mobility solutions that are consumed as a service. Transport information together with telecommunications infrastructures allows developing real-time systems that convert mobility into a service.

In addition, to fulfilling the purpose of this framework, the embedded simulation tool must have certain capabilities, namely that it must be able to:

- Represent the road network geometry in detail.

- Realistically emulate the time evolution of traffic flows on the road network. Vehicles in the simulation model travel from origins to destinations along the corresponding paths while taking into account the time.

- Deal with multiple vehicle classes with differentiated behaviours in order to account for the specific characteristic of the vehicles in the fleets being studied.

- Provide a real-time interface access during the simulation. These libraries should offer information relative to the vehicles, nodes and arcs in the graph that represent the network topology, traffic lights, computed paths, etc. This would allow implementing the aforementioned functions and modules when addressing different case studies. Furthermore, it is also interesting that the simulator can interact with external applications, by using either these very same interfaces or any other method.

Different network representations and simulation approaches have been used: mesoscopic and microscopic. One of the two will be selected according to the specific necessities and expectations of the project. Macroscopic models could also be incorporated into the platform.

The current implementation of this framework includes Aimsun [14] as a traffic simulation tool, although this selection could easily be changed. The exchange of information between the external application and the simulator is made at every simulation step, which is the time interval at which the estate of the simulation model is updated. The programming languages in which Aimsun provides its API are C++ and Python. While Python is used to easily collect some of the data, $\mathrm{C}++$ is needed for the emulation of the different applications due to performance reasons.

\section{Execution Controller}

An experimental simulation consists of a certain predefined number of replication executions. These replicas are launched from a controller, and a pre-process sets each of them up. At the end of a simulation, a post-process is run in order to collect the data generated from it.

- Controller: responsible for launching the simulator. In addition, it manages the directory tree where the execution results are stored along with a history of all the executed simulations.

- Pre-run process: computes the previous calculations of items, such as demand matrices, vehicle type configuration and others that depend on the experiment's specific requirements.

- Post-run process: during this process, the selected KPIs are extracted from the output databases generated by the simulator. Additionally, other data required for the visual analytics features is also collected (section and turning information, etc.).

\section{Results Processing module}

The Results Processing module [15] covers the automatic collection and pre-processing of the data before its visualization. This module is composed of an Extract-Transform-Load process (ETL Process), which is responsible for finding new execution results from recently performed simulations and processing them for the Visual Analytics module.

These results are stored on a server and are obtained from two types of files:

- SQLite databases, which are standard outputs from the Aimsun simulator.

- CSV files, which are generated by specifically developed APIs during the executions of the replications. These depend on the application that is being emulated. 
Specifically, the ETL Process refers to extracting, transforming and loading the results of the traffic simulation executions. In particular, it performs the following actions:

- Load new simulation replication results.

- $\quad$ Store replication KPIs that the simulator provides by default and which are needed for analysing the results.

- Calculate and store replication KPIs that the simulator does not provide by default. (For instance, flow by lane).

- Calculate and store global KPIs for each simulated scenario.

The output of the ETL Process is a set of RDS files (internal single object files) in an R [16] data frame-like format, which allows enhancing the subsequent access speed. This improvement is possible because the Visual Analytics module does not need to directly access and process the data from SQLite or the CSV files each time they are generated by the simulation executions. In fact, this pre-process reduces the time by $99.5 \%$ in comparison with direct access [15].

\section{Visualization and analytics}

The Visual Analytics module in Figure 3 is based on Lorente [17] and covers the display of the simulation results from any previously defined replication execution. In addition, it allows for the visualization of input data and other simulation model details.

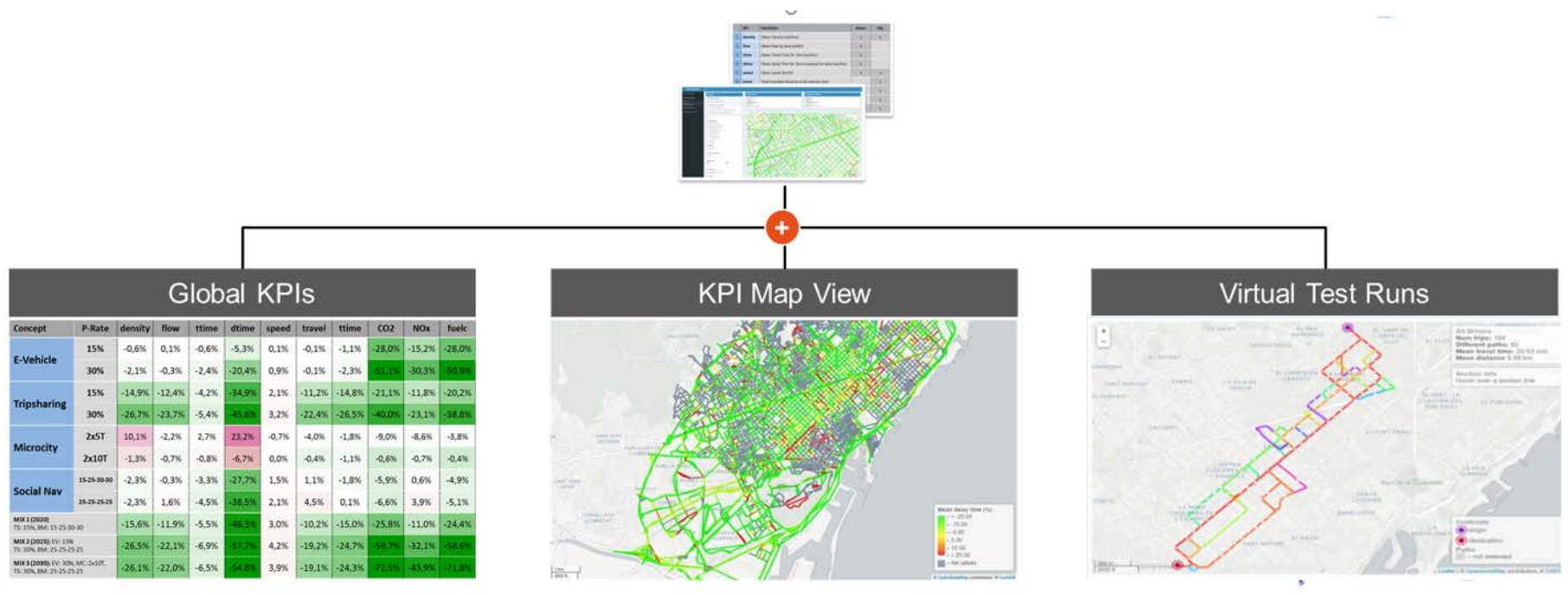

Fig. 3. Overview of CitScale's Visualization possibilities.

The development of this module includes two different components:

- The visualization application that is implemented with $\mathrm{R}$ in the framework of Shiny [18], which allows for development without directly using web code languages.

It is important to note here that, for performance reasons, the C++ development language is also used in some of the application computations. Furthermore, for greater efficiency in some cases, certain processes are implemented directly with HTML, CSS and JS.

- $\quad$ Direct user access to the server hosting the application. The Shiny server's functionalities facilitate this process.

The Visual Analytics module provides different kinds of analysis for evaluating traffic simulation results, depending on the case study.

First, the case study must be selected using the CitScale platform, as shown in Figure 4. 

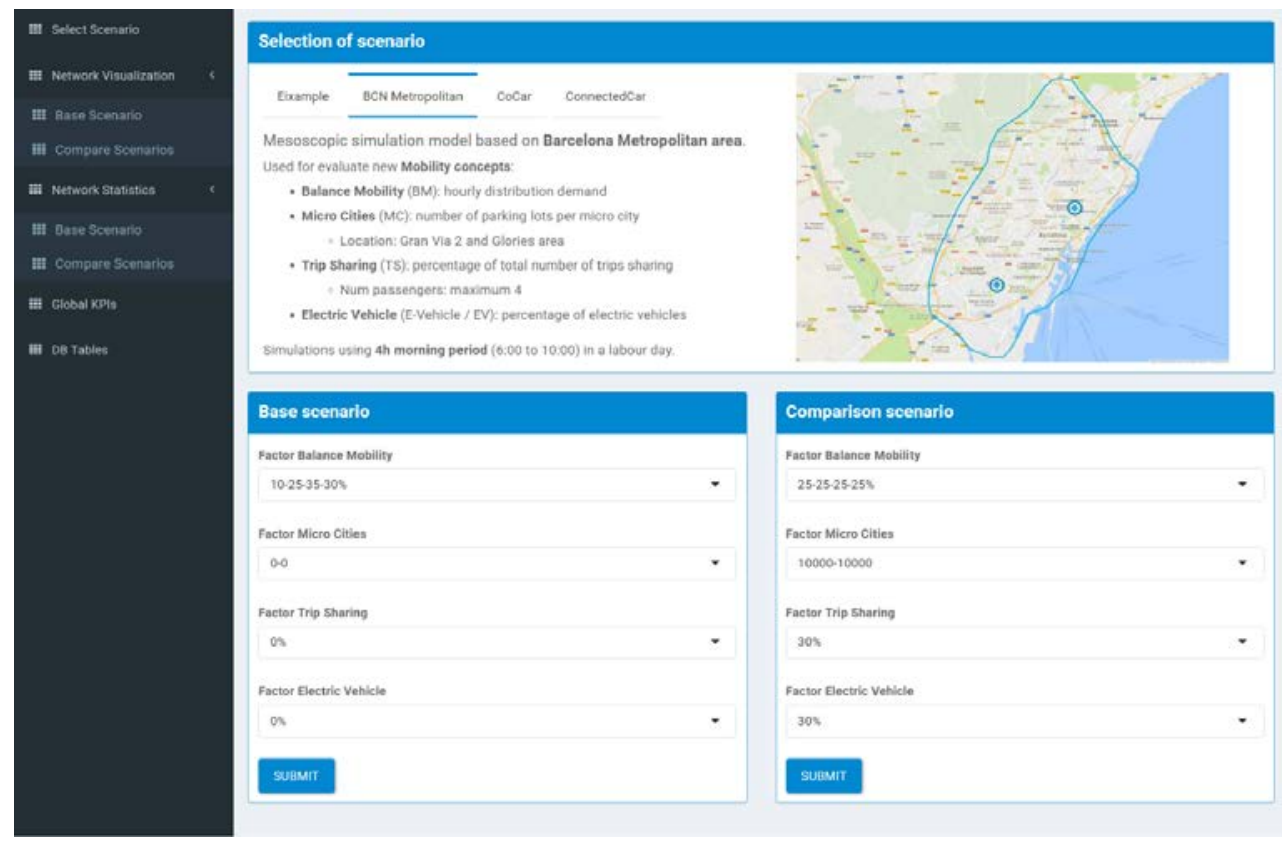

Fig. 4 Case Study Selection on the CitScale Platform.

Traffic simulation results depend on the simulation study, because these results depend on the traffic assignment tool that was selected in the experimental design for the study's simulation engine. Whether they are graphic or numeric, KPI Analytics are available for all case study scenarios. For comparison, a base scenario and an alternative scenario must be selected. In the lower part, different drop-down menus are available for changing the base/comparison scenario configurations. These drop-down menus allow choosing from among all possible configurations for the experimental design. The KPIs are obtained by combining the results from all replications of each configuration. The following provides an outline of the analytical capabilities based on the case studies analysed through the CitScale platform. Four simulation projects have been incorporated into the CitScale platform as case studies: Eixample, Metropolitan, CoCar and Connected Car. The Eixample, CoCar and Connected Car cases rely on Aimsun microscopic simulation modelling for Barcelona city's Central Business District (CBD), which is called the Eixample; while the Metropolitan case study relies on a mesoscopic simulation model for the Inner Ring Road area of Barcelona's Metropolitan Area, which includes the city of Barcelona and parts of the metropolitan municipalities L'Hospitalet, Esplugues, Cornellà, Sant Adrià and Santa Coloma.

The Key Performance Indicators that can be analysed on a time-dependent basis are:

- Total Travelled Distance $(\mathrm{km})$.

- Total Travelled Time (h).

- $\quad$ Fuel - Total litres of fuel consumed.

- $\quad \mathrm{CO} 2(\mathrm{~kg})$.

- $\quad \mathrm{NOx}(\mathrm{kg})$.

- Average Travel Time (sec/km).

- Mean Delay time (sec/km).

- $\quad$ Density (veh/km).

- $\quad$ Average speed $(\mathrm{km} / \mathrm{h})$.

- Average Flow (veh/h).

- $\quad$ Throughput rate (\%) completed trips divided into total number of trips for $1 \mathrm{~h}$.

\section{Eixample Case Study}


A microscopic simulation [19] approach was chosen for evaluating the selected scenarios in the case study's experimental design. This allows us to quantify the impact of vehicle concepts such as electric bikes (E-Bikes), Last Mile Surfer (LMS) and electric vehicle (E-Vehicle) penetrations [20]. A brief description of the visualization possibilities can be found in the following:

- Global KPI analysis: This component shows different summary tables for all the simulation scenario results from each particular experimental design (Figure 5). The first table displays the global values for each defined KPI and scenario, and the two additional tables display relative and absolute differences between each of the base scenario configurations.

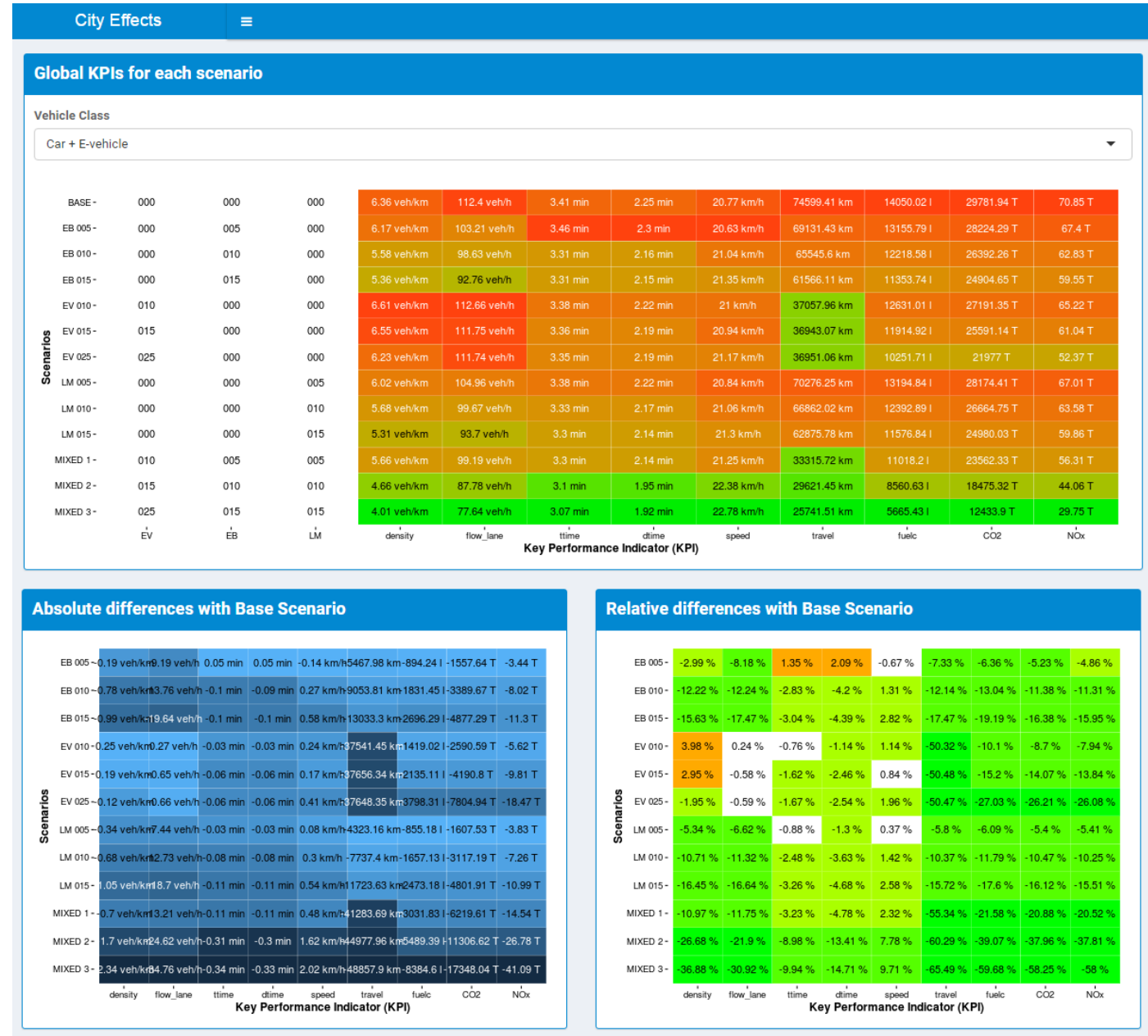

Fig. 5 Example of Global KPI Table Visualization.

- City network impact: This analysis allows visualizing the KPI results of a simulation replica by superimposing it over the current network model. It shows the selected KPI values at the link level for each simulation interval ( $3 \mathrm{~min}$ ) and the overall simulation horizon (1h). In Figure 6, a fuel consumption KPI plot for a $1 \mathrm{~h}$ time horizon is indicated by a particular colour, depending on the platform-defined scale. On the left hand side, filters and KPI selector are available. A visual comparison of scenarios is shown in Figure 7. Relative/absolute comparison functions can be selected via drop-down menus. Specifically, this allows us to visualize the fuel consumption KPI comparison for each simulation interval or, alternatively, an animation allows us to evaluate the temporal evolution of the KPIs for any given scenario comparison. Also, the histogram on the top right side gives an idea of how the KPI comparison behaves over time. 


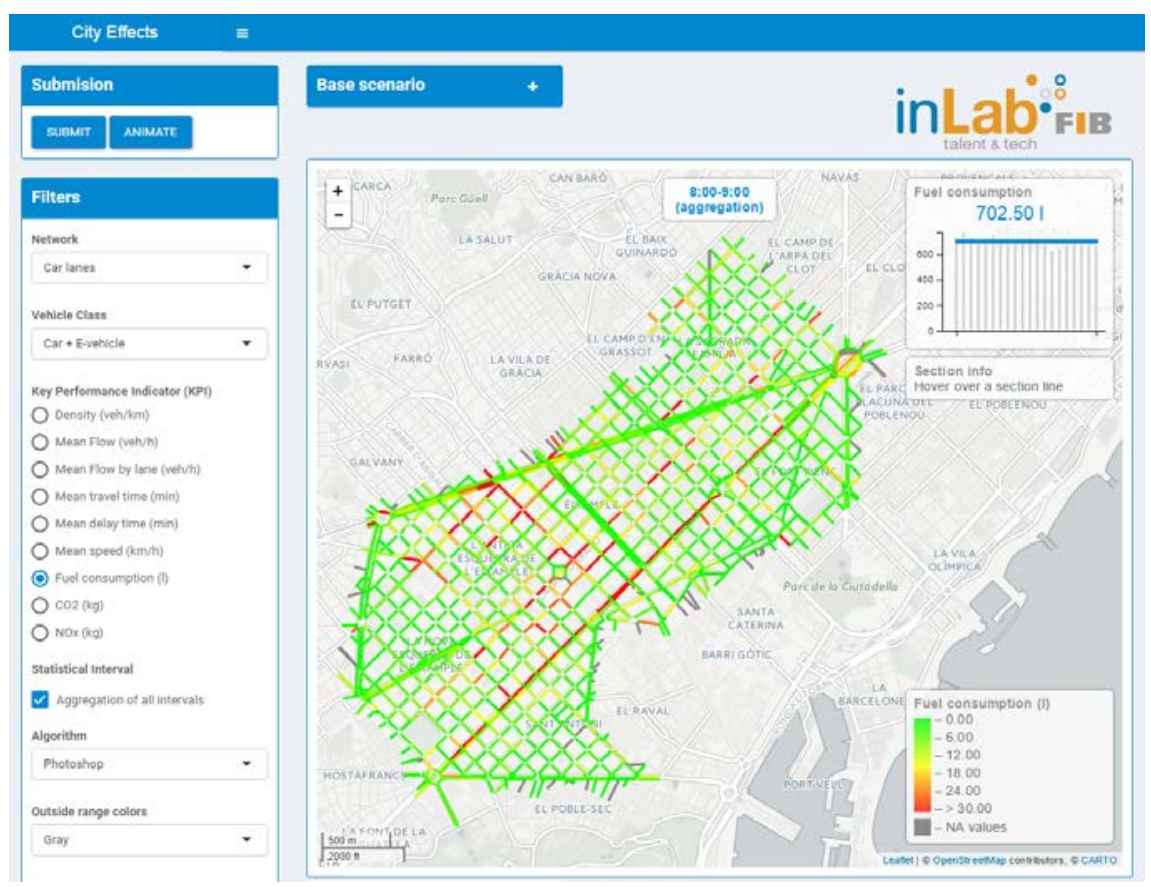

Fig. 6 Barcelona's CBD Base Scenario: KPI Network Impact Visualization.

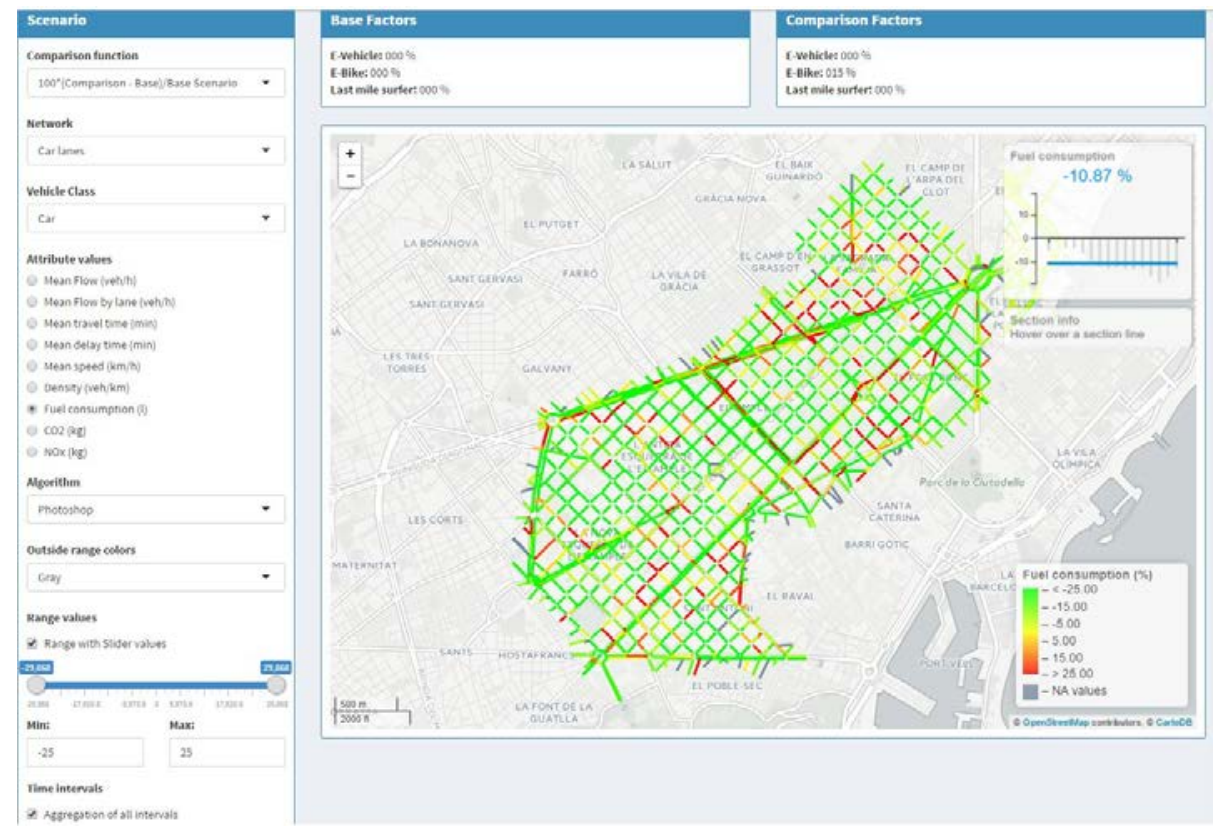

Fig. 7 Barcelona's CBD Scenarios for comparison of KPI Network Impact Visualization.

- Analysis of routes used: This analysis allows visualizing all the paths used in a simulation replica on a timedependent basis. Selecting the specific vehicle class (regular car, e-vehicle, e-bike, LMS) is allowed in the dropdown menu. Either Auto or Bike network can be selected. A line-path visualization renders all the streets included in the origin-destination paths for each vehicle class (Figure 8). This particular analysis can be performed on Barcelona's CBD case study because a microscopic simulation approach allows for individual vehicle tracking and obtaining vehicle class origin-destination path statistics. The road segments used on paths from origin to destination centroids (OD paths) and all vehicle classes are shown in the Fig. 8 visualization. Specifically, this visualization shows OD paths using an opacity scale according to the OD path flows (i.e., the more opaque it is, the greater the flow of vehicles along the 
road segments on the OD path). In addition, the right side provides some information on all road segments and their statistics, as well as the legend and individual information on each section.

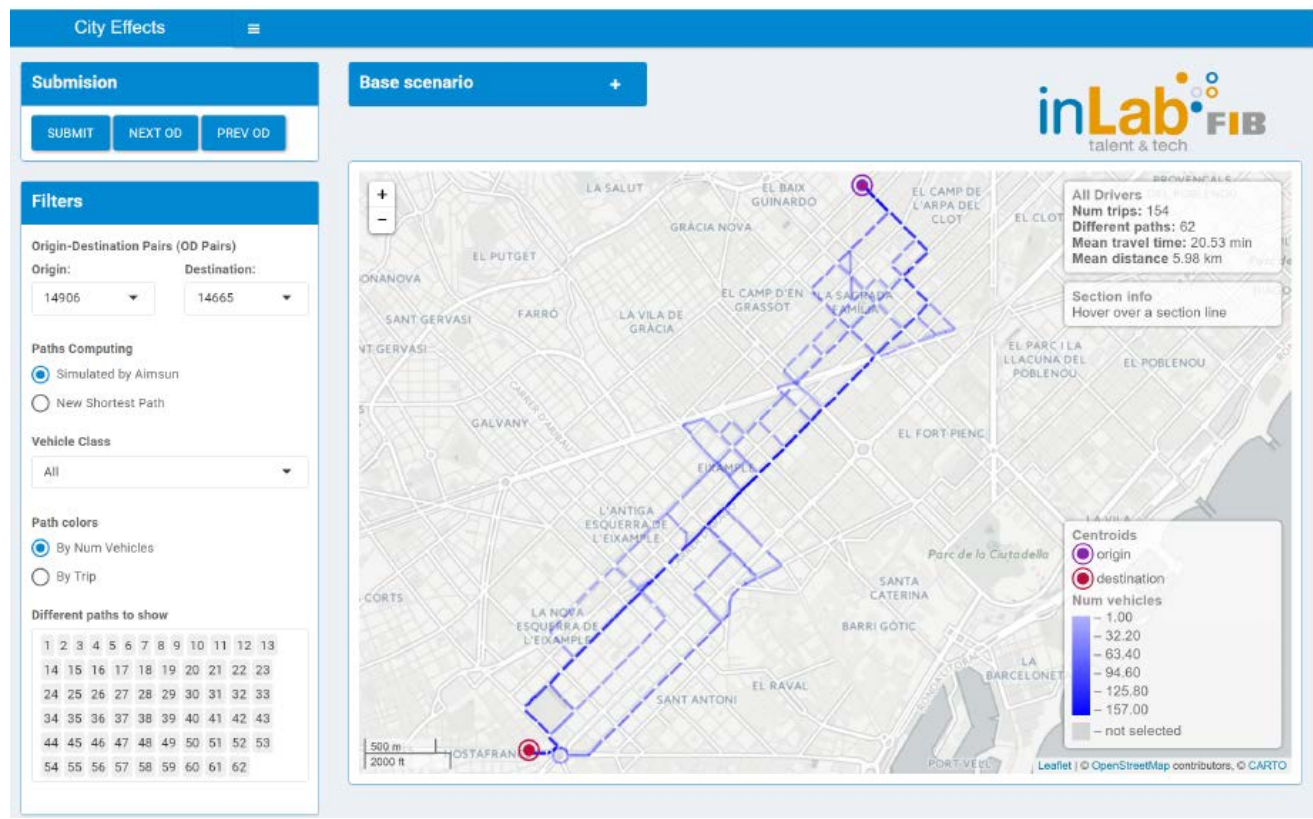

Fig 8. Example of Trips Visualization.

- Mobility patterns: This feature shows a visualization and analysis of the overall horizon mobility patterns (origins and destinations of the trips). The trips among origin-destination pairs are displayed, as well as the demand generation and attraction of each centroid. This display is shown at the vehicle class level and for the whole metropolitan area. Remarkable desire lines (OD relations with remarkable number of trips) are clearly highlighted (Figure 9). Filtering OD pairs according to the number of trips or OD trip length (Euclidean distance between centroids) are also allowed. A histogram of the active number of OD trips is displayed in the upper right visualization.

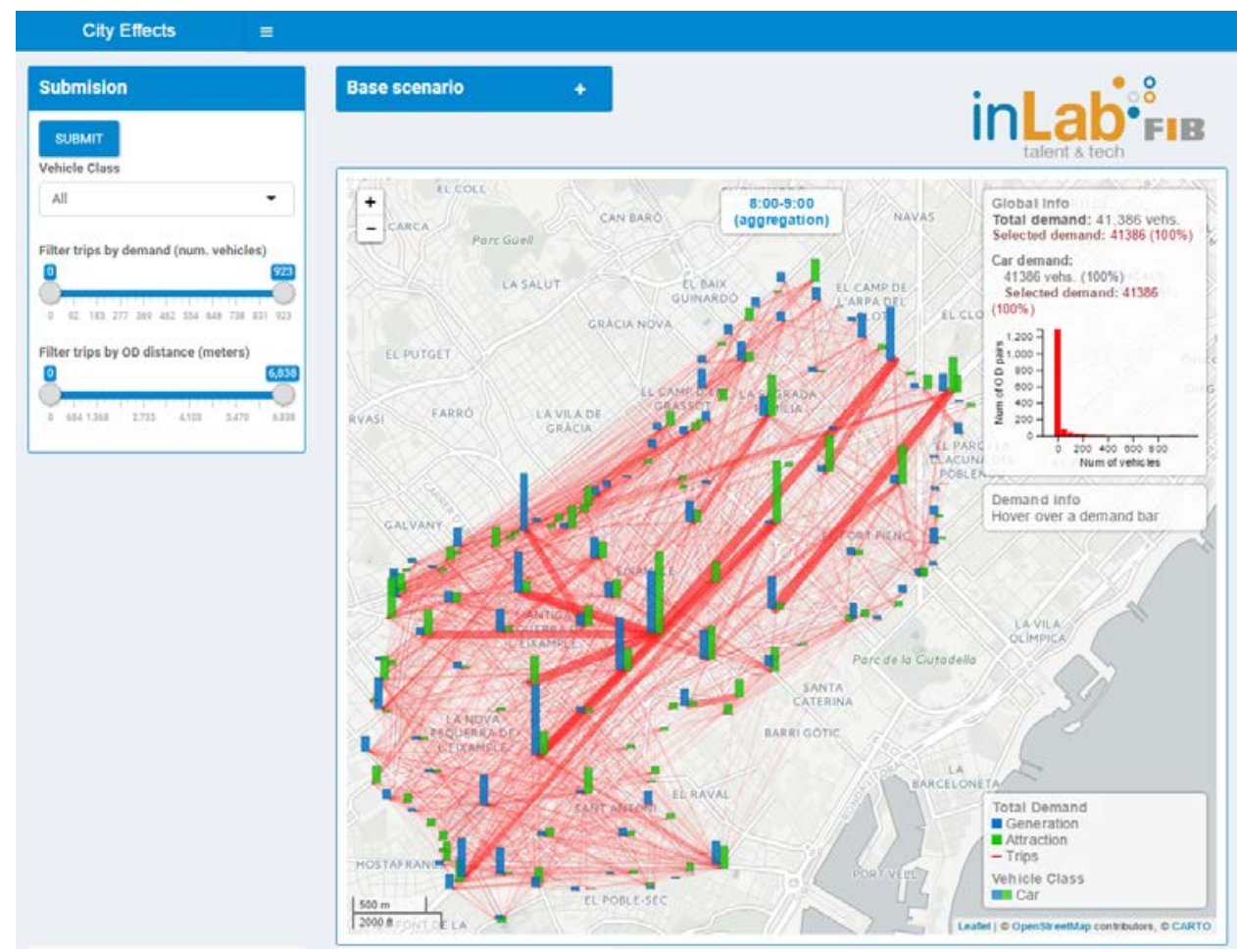

Fig 9. Example of Mobility Pattern Visualization. 


\section{Metropolitan Case Study}

A mesoscopic simulation approach was selected for the metropolitan [11] scenario because the dimensions involved in executing microscopic simulation replicas would make the computational burden. The inner ring part of the Barcelona metropolitan area was considered in the traffic model for this project and subsequent visual analytics. It comprises $102 \mathrm{~km}^{2}$ and more than $1,700,000$ inhabitants. The model of the inner rings area consists of 16,000 links, 8,000 nodes and a horizon study of $4 \mathrm{~h}$, accounting for a total number of 350,000 vehicle trips. This case study addresses:

- Vehicle concepts related to electric vehicle penetration (10\%-25\%-35\%-30\% levels)

and mobility concepts such as:

- The impact of 2 new hubs defined across the area (issues regarding trip distribution changes were defined for the origindestination demand matrices that are the input to the mesoscopic simulation engine). The aim of Microcities is to reduce the number of trips that arrive at their final destination by car, leading to fewer emissions and less congestion. The total trip attraction in target areas is proportionally increased by the additional parking capacity (either 1,500 trips-5,000 lots, or 3,000 trips when 10,000 lots per parking are considered). Thus, former destinations are reduced in affected zones for trips that consider parking at new hubs. Microcities Mesoscopic modelization is affected by 2 parameters:

o Microcity location. Gran Via 2 and the Glòries shopping areas.

o Microcity capacity (number of parking lots). We consider a large number of either 5,000 or 10,000 lots.

- Tripsharing penetration scenarios. The aim of trip sharing is to reduce the number of cars in the network; thus, fewer emissions and less congestion must be observed. Trip sharing Mesoscopic modelization is affected by 3 parameters:

o Percentage of the total number of trips suitable for TripSharing (tested levels $0 \%, 15 \%$ and $30 \%$ ).

o Maximum number of passengers for shared vehicles (selected max 4 pax/car).

o Interval length $\mathrm{T}$ for grouping common trips (a 15-min window is selected): only trips with the same origin and destination and starting time are considered for trip-sharing purposes.

- Starting trip time share for OD trips over hours involved in the time-horizon (4h): $10 \%, 25 \%, 35 \%$ and $30 \%$ hourly distribution was set as the reference level (current situation in the study area); while 3 alternative levels were also addressed, one of them being the full-balance situation $25 \%-25 \%-25 \%-25 \%$.

Fig. 10 on the left shows the status of each selected road segment with respect to the selected KPI. The comparison function of the KPI between the two scenarios is shown in the right-hand graph. This last visualization allows evaluating which of the two scenarios gives better results. This analysis can be seen from two points of view:

- By comparing a new scenario with the base scenario (which represents the current situation, in general), it is possible to evaluate whether the new situation improves the state of the road network. Specifically, the selected KPI is contrasted with the base scenario.

- By comparing two new scenarios based on different concepts, one can evaluate which of the two is better.

In addition, an animation can be displayed to illustrate either: the evolution of the selected KPI over each time interval (15 min) in the defined time horizon ( $4 \mathrm{~h}$ for this case study); or the aggregated results for the overall time horizon (4h).

The drop-down menu on the left banner allows us to select the vehicle class (either regular car or e-vehicle in the current study) and auto network (car lanes option). These are the only possibilities, as bikes cannot be modelled by mesoscopic simulation. 

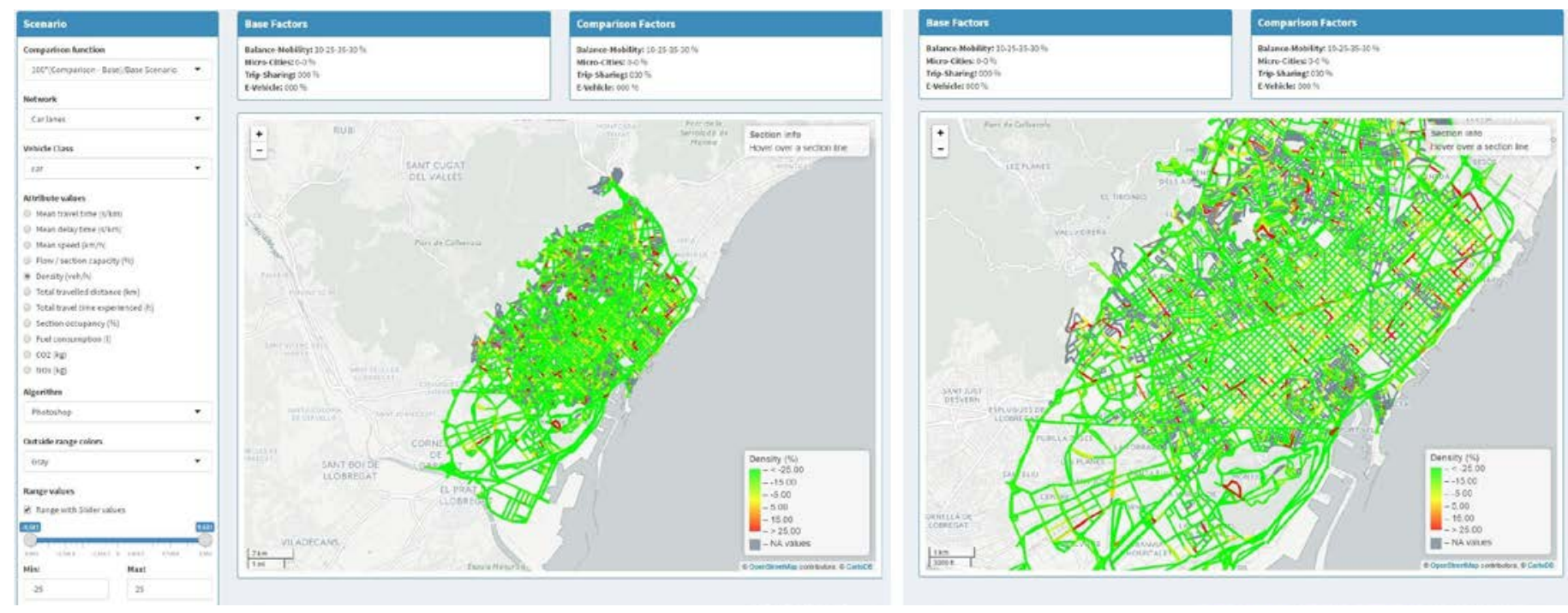

Fig 10. Metropolitan Case Study: KPI Visualization. Left: basic scenario. Right:Comparison of 2 scenarios.

An ad-hoc visualization for CO2 emissions KPIs was included, as shown in Figure 11.

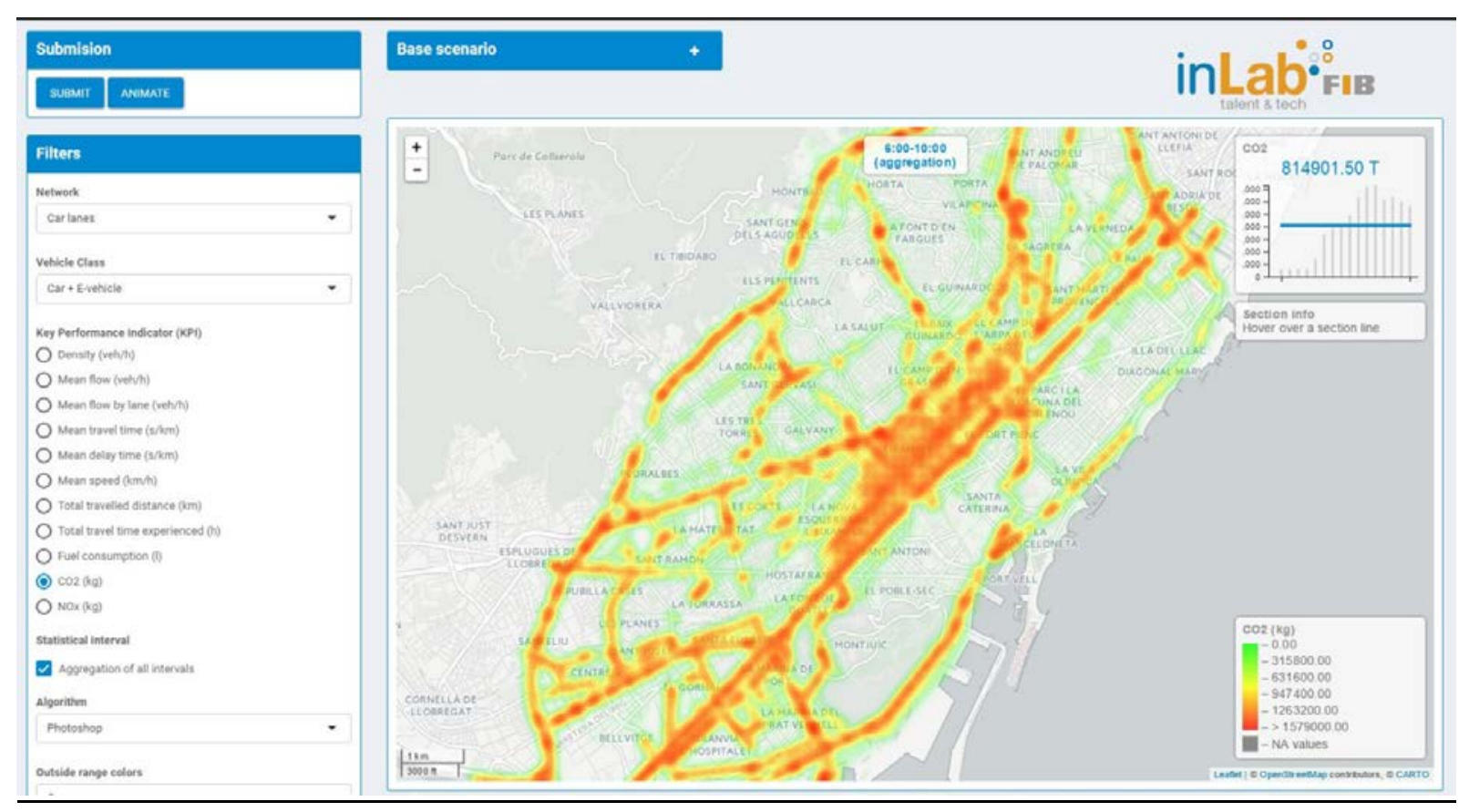

Fig 11. Metropolitan Case Study: CO2 Visualization.

The conclusions drawn from the KPI analysis through CitScale are:

- As trip sharing penetration increases, average travel time for cars is slightly reduced and delay times are strongly reduced.

- $\quad$ As trip sharing penetration increases, average speed slightly increases by between $2 \%$ to $3.5 \%$.

- As trip sharing penetration increases, fuel consumption and emissions KPIs are strongly reduced at the same rate, plus an extra rate.

- $\quad$ As e-Vehicle penetration increases, traffic KPIs for cars become stable. 
- As e-Vehicle penetration increases, fuel consumption and emissions KPIs reduce at the same rate (fuel), plus an extra rate (CO2 and NOx).

- As Balanced Mobility changes, the demand becomes more homogeneous and the effect seems to be a slight improvement in almost all the KPIs.

- The demand pattern is modified with Microcities and no significant differences are found in the global KPIs. Differences can be found as local effects in the map representation.

\section{CoCar Case Study: Multiple Passenger Ride Sharing}

The objective of this case study based on simulation is to evaluate a new mobility concept under the MaaS paradigm: multiple passenger trip-sharing [21], [22]. This describes a shift from personally owned modes and towards mobility solutions as a service. Transport information, together with telecommunications infrastructures, allows for the development of real-time systems for converting mobility into a service.

In order to extend the standard functionalities of the selected simulation tool so that it can deal with the specific requirements of this case study, innovative mobility concepts were incorporated into the Custom Module (Figure 2). The aim of trip sharing is to reduce the number of cars in the network, thus producing fewer emissions and less congestion.

- CoCar Vehicle characteristics were assumed to be: a length of $5.0 \mathrm{~m}$ and width of $2.10 \mathrm{~m}$, both fixed.

- Car-following and lane-changing driving behaviour parameters

o Desired speed fixed at $50 \mathrm{~km} / \mathrm{h}$

o Full speed acceptance at segments, no variability considered

o Reaction time between $0.8 \mathrm{sec}$ and $1.2 \mathrm{sec}$ at stops.

- Inter-vehicular distance: $1 \mathrm{~m}$, no variability considered

- Route Choice selection: Vehicle Routing algorithms specifically for serving trip requests

- No Origin-Destination data for fleet units

- Network use: regular and bus reserved lanes

- Stops: At bus stops and specific locations at chamfered intersections (known locally as chafláns)

- Demand penetration scenarios of $5 \%, 10 \%$ and $15 \%$ of total autos during the morning period.

The system was designed to be flexible in order to host a wide range of service possibilities by setting the values of parameters determining the behaviour of the system, such as:

- $\quad$ Fleet size, vehicle capacity, and the market quota for the new mode as a percentage of total passenger car demand for the study period

- $\quad$ Fully flexible routes, fixed routes or mixed routing

- Boarding/Alighting either everywhere in the network, at pre-defined stops, regular bus stops, or any chamfered intersection in the Eixample geometry

- Depot existence: number and location

- Pre-booked demand service available or not

- Combined service for passenger and freight distribution

- Use of reserved bus lanes

The factors considered in the experimental design are:

- Capacity of vehicles operating in the new model: 6, 8 or 10 passengers

- Fleet dimension: $250,500,750$ or 1000 vehicles. 
- Time windows in which the demand requests must be served (pick-up and delivery): 10, 15 or 30 min, or without constraint.

- The percentage of total demand for the study period that is not pre-booked in advance: $0 \%, 20 \%, 50 \%, 80 \%$ and $100 \%$.

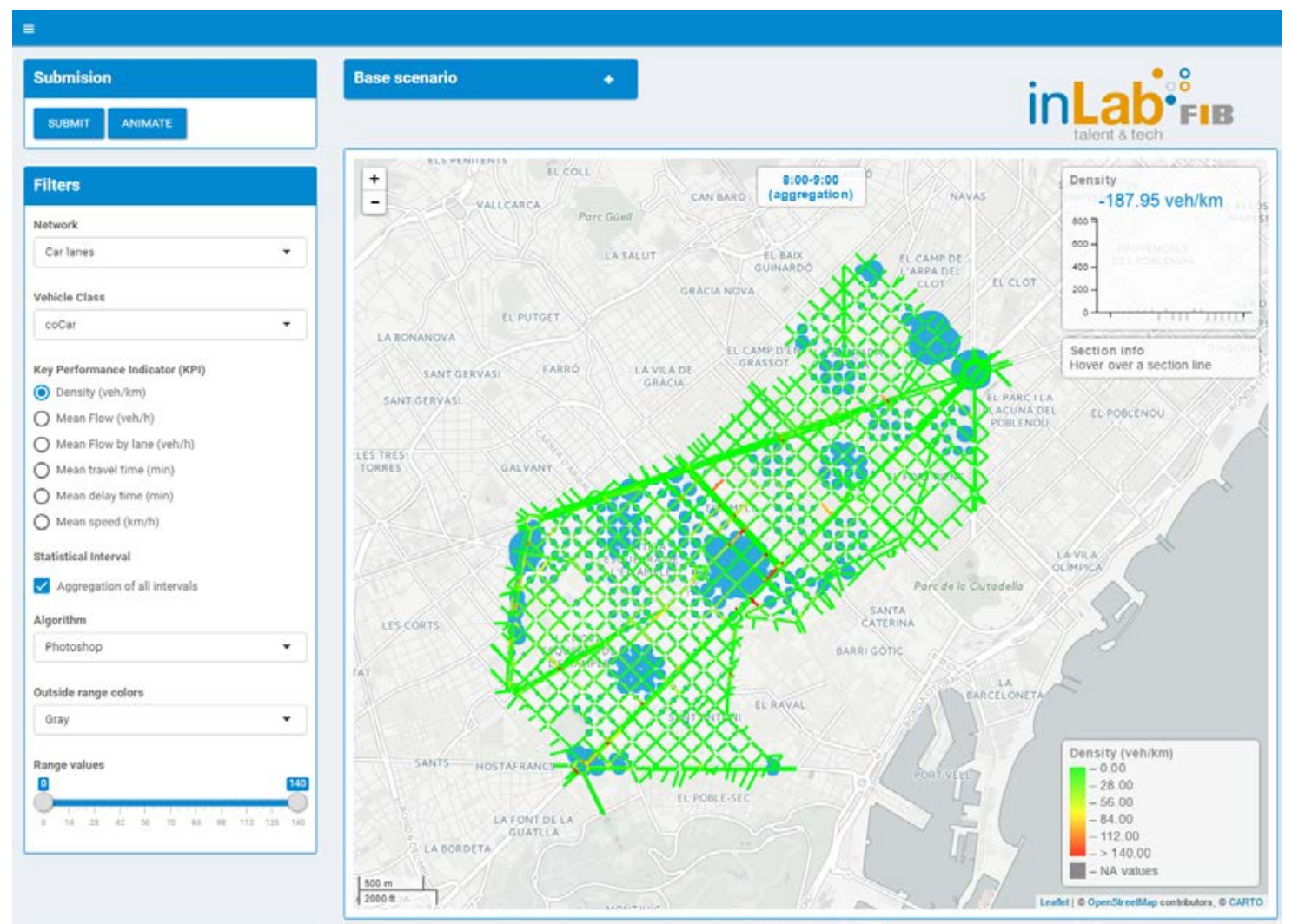

Fig 12. City networks impacts (density) and car-sharing patterns.

Figure 12 shows the city network impacts of the CoCar system and the proposed demand generation patterns. The key conclusions drawn from the results after executing the microsimulation CoCar case study in CitScale tool are:

- Fleet size has an important effect on the percentage of served demand requests. With a total demand request of $10 \%$ of the private vehicle demand (approx. 3.300), with 500 vehicles in the fleet, the CoCar system can serve approximately $78 \%$ of the requests. With 750 vehicles, the percentage increases to $90 \%$. With 1000 vehicles, the percentage is practically the same.

- The median occupancy is 2.14 pax/vehicle.

- The average service time decreases when the percentage of dynamism in the demand request decreases.

- The time window factor has a significant effect on the average service time. It explains nearly $80 \%$ of the total variability in service time.

\section{Connected Car Case Study}

This case study shows how the traffic data on Connected Car penetration [19], [20], [23] could improve existent navigation strategies for guided vehicles and lend support in reducing city-wide network congestion effects. Drivers were divided into Guided and Non-Guided for each of the three vehicle classes, defined according to driving behaviour: tourist (low network and congestion knowledge); regular drivers (knowledge of congestion for daily-used routes); and Expert drivers (level of congestion known for 
routes). Comparative analysis of navigation strategies (lane-based vs. link-based) was also addressed. The design of experiments for microsimulation modelling was composed of seven factors, each defined by 2 to 5 levels:

- $\quad$ Factor A - Type of drivers, i.e. Expert, Regular and Tourist (triplets E-R-T). This affects car-following, lane changing and gap acceptance model parameters. Route choice behaviour is also type dependent. Considered Levels: 40-50-10 (ref), 090-10 and 20-70-10.

- Factor B - Navigation Penetration: Percentage of the total vehicles following Route Guidance Mobility Service. Considered levels: 5\%, 10\% and 20\% (ref). A 100\% Guidance Acceptance was also considered.

- Factor C - Demand Pattern, Historically Based Increments: Increments in the historical patterns of the long trip segment. Considered Levels: 0\%, 20\% (ref).

- $\quad$ Factor D - Connected Car Penetration (CCP): Penetration rate of the total number of probe cars (equipped with GPS tracking). Considered Levels: $0 \%, 10 \%, 20 \%, 30 \%, 100 \%$ and Perfect Info (ref). Traffic variables derived from equipped cars depend on estimators: speed, density, flow, speed in motion, queue length, percentage of stop time, etc.

- Factor E-Time-window: length of time necessary for using Connected Car data to estimate traffic variables. Considered Levels: $3 \mathrm{~min}$ (ref) and $6 \mathrm{~min}$.

- $\quad$ Factor F - Navigation Strategy: Considered levels: (1) Link level Navigation, (2) Lane level Navigation.

\begin{tabular}{|c|c|c|c|c|c|c|c|c|c|c|c|c|c|c|}
\hline Drivers & $\begin{array}{c}\text { Navigation } \\
\text { Penetration }\end{array}$ & $\begin{array}{c}\text { Demand } \\
\text { Pattern }\end{array}$ & $\begin{array}{c}\text { Connected } \\
\text { Car } \\
\text { Penetration }\end{array}$ & $\begin{array}{c}\text { Time } \\
\text { Window }\end{array}$ & $\begin{array}{c}\text { Navigation } \\
\text { Strategy }\end{array}$ & $\begin{array}{c}\text { Density } \\
\text { (veh/km) }\end{array}$ & $\begin{array}{c}\text { Mean Flow } \\
\text { (veh/h) }\end{array}$ & $\begin{array}{c}\text { Mean } \\
\text { Travel Time } \\
(\mathrm{sec} / \mathrm{km})\end{array}$ & \begin{tabular}{|c|} 
Mean \\
Delay Time \\
$(\mathrm{sec} / \mathrm{km})$
\end{tabular} & $\begin{array}{l}\text { Mean } \\
\text { Speed } \\
(\mathrm{km} / \mathrm{h})\end{array}$ & $\begin{array}{c}\text { Total Travelled } \\
\text { Distantance } \\
(\mathrm{km})\end{array}$ & $\begin{array}{c}\text { Fuel } \\
\text { Conumption } \\
\text { (L) }\end{array}$ & $\mathrm{CO} 2(\mathrm{~kg})$ & NOx (kg) \\
\hline $40-50-10$ & 20 & 20 & 0 & 3 & Link Navigation & $0,00 \%$ & $0,00 \%$ & $0,00 \%$ & $0,00 \%$ & $0,00 \%$ & $0,00 \%$ & $0,00 \%$ & $0,00 \%$ & $0,00 \%$ \\
\hline $40-50-10$ & 20 & 20 & 10 & 3 & Link Navigation & $-4,53 \%$ & $1,67 \%$ & $-5,48 \%$ & $-7,89 \%$ & $1,17 \%$ & $3,14 \%$ & $1,47 \%$ & $0,41 \%$ & $0,11 \%$ \\
\hline $40-50-10$ & 20 & 20 & 20 & 3 & Link Navigation & $-6,64 \%$ & $3,07 \%$ & $-6,33 \%$ & $-9,12 \%$ & $1,42 \%$ & $4,75 \%$ & $2,51 \%$ & $0,44 \%$ & $0,17 \%$ \\
\hline $40-50-10$ & 20 & 20 & 30 & 3 & Link Navigation & $-5,66 \%$ & $1,94 \%$ & $-7,28 \%$ & $-10,47 \%$ & $2,53 \%$ & $3,63 \%$ & $0,80 \%$ & $-0,26 \%$ & $-0,43 \%$ \\
\hline $40-50-10$ & 20 & 20 & 100 & 3 & Link Navigation & $-9,15 \%$ & $3,30 \%$ & $-9,12 \%$ & $-13,17 \%$ & $3,32 \%$ & $4,96 \%$ & $1,08 \%$ & $-0,95 \%$ & $-1,45 \%$ \\
\hline $40-50-10$ & 20 & 20 & Perfect Info & 3 & Link Navigation & $-8,56 \%$ & $2,86 \%$ & $-7,87 \%$ & $-11,38 \%$ & $2,96 \%$ & $4,33 \%$ & $0,61 \%$ & $-1,29 \%$ & $-1,65 \%$ \\
\hline $40-50-10$ & 20 & 20 & 0 & 3 & Lane Navigation & $-5,08 \%$ & $0,96 \%$ & $-4,01 \%$ & $-5,80 \%$ & $1,74 \%$ & $1,23 \%$ & $-0,98 \%$ & $-1,70 \%$ & $-1,86 \%$ \\
\hline $40-50-10$ & 20 & 20 & 10 & 3 & Lane Navigation & $-8,14 \%$ & $3,30 \%$ & $-8,34 \%$ & $-12,07 \%$ & $2,75 \%$ & $5,69 \%$ & $2,22 \%$ & $0,10 \%$ & $-0,12 \%$ \\
\hline $40-50-10$ & 20 & 20 & 20 & 3 & Lane Navigation & $-10,33 \%$ & $3,76 \%$ & $-9,67 \%$ & $-13,97 \%$ & $3,70 \%$ & $6,04 \%$ & $1,61 \%$ & $-0,89 \%$ & $-1,31 \%$ \\
\hline $40-50-10$ & 20 & 20 & 30 & 3 & Lane Navigation & $-9,80 \%$ & $2,48 \%$ & $-11,17 \%$ & $-16,13 \%$ & $4,63 \%$ & $4,43 \%$ & $-0,87 \%$ & $-2,04 \%$ & $-2,23 \%$ \\
\hline $40-50-10$ & 20 & 20 & 100 & 3 & Lane Navigation & $-12,85 \%$ & $4,63 \%$ & $-11,61 \%$ & $-16,79 \%$ & $4,90 \%$ & $6,77 \%$ & $1,25 \%$ & $-1,90 \%$ & $-2,45 \%$ \\
\hline $40-50-10$ & 20 & 20 & Perfect Info & 3 & Lane Navigation & $-11,58 \%$ & $3,75 \%$ & $-10,80 \%$ & $-15,60 \%$ & $4,54 \%$ & $5,70 \%$ & $0,31 \%$ & $-2,02 \%$ & $-2,47 \%$ \\
\hline
\end{tabular}

Fig. 13 Comparative Global CitScale KPIs - Connected Car Case Study

Figure 13 shows a table of comparative Global CitScale KPIs per experimental unit ( 5 replicas) with respect to the basic scenario (the one with reference levels, as described in the previous list). CitScale allows us to visualize the routes used by each vehicle class, in this case defined by 6 categories - which were obtained by crosschecking Guided/Non-Guided attributes with Driver Behaviour (Tourist, Regular and Expert). Figure 14 shows the OD pair (drop-down menu selection) routes used by the Tourist-Non-Guided vehicle class. It also indicates the number of different OD paths, mean OD travel time, distance for the selected vehicle class, and a comparison with all driver statistics. 


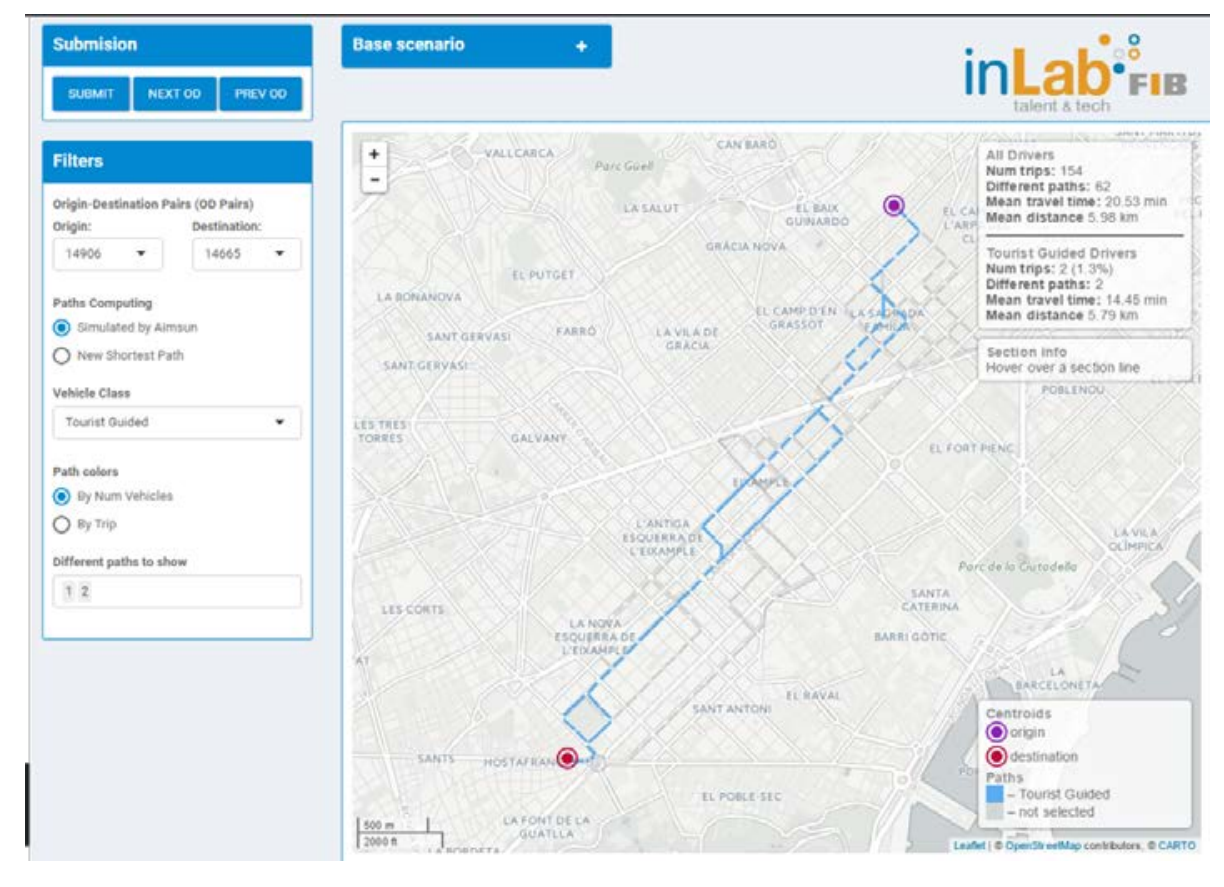

Fig. 14 Vehicle Class Route Analysis: CitScale - Connected Car Case Study

Conclusions drawn after loading the microsimulation execution results into CitScale are:

- The trip analysis shows that there are more trips at low speeds in Non-Guided and Link Navigation strategies.

- $\quad$ From a city perspective: density, mean travel time, mean delay time and mean speed are better with higher levels of Connected Car penetration. Lane Navigation values are slightly better than Link Navigation values. The reduction in $\mathrm{CO} 2$ and NOx follows the same pattern but at lower values. Travelled distance is greater with more CCP, and slightly greater with Lane Navigation rather than with Link Navigation.

- Mean travel time is lower with higher levels of CCP, and better with Lane Navigation than with Link Navigation.

- Mean travel distance and mean speed are greater with higher levels of CCP, and Lane Navigation obtains better results.

- In comparing the types of drivers (Tourist, Regular and Expert), Link and Lane navigation provides better reduction in travel time for Tourists and less reduction for Experts. The reduction in travel time is slightly better with Lane Navigation.

The CitScale visualization and analytics platform has proven to be very practical in supporting the results analyses for different experimental designs. The designs applied here incorporated different traffic simulation models for various purposes. A project for Probe Vehicle Data assessment has been incorporated into CitScale, as well as another project that focuses roughly on analysing the impact of emissions in the Eixample on KPIs, specifically when the city shifts to more green modes such as bicycles or electric vehicles. A further development for improving demand modelling in CitScale will provide representations of activity-based demand. This flexible tool will save a lot of time for traffic analysts and future transportation projects at the UPC.

\section{Acknowledgements}

This research has been funded by TRA2016-76914-C3-1-P of the Spanish R+D National Programs and by the Secretaria d'Universitats i Recerca de la Generalitat de Catalunya under 2017-SGR-1749. Throughout, the authors have benefited from the support of inLab FIB (UPC).

\section{References}


[1] P. Rode, C. Hoffmann, J. Kandt, A. Graff, and D. Smith, "Towards new urban mobility: the case of London and Berlin," The London School of Economics and Political Science, LSE Cities, 2015.

[2] M. Furuhata, M. Dessouky, F. Ordóñez, M.-E. Brunet, X. Wang, and S. Koenig, "Ridesharing: The state-of-the-art and future directions," Transp. Res. Part B Methodol., vol. 57, pp. 28-46, Nov. 2013.

[3] P. Basnal, V. Singh, A. Shukla, D. Kumar, and A. Kadam, “Demand Responsive Transport," IJISET - Int. J. Innov. Sci. Eng. Technol., vol. 2, no. 4, 2015.

[4] International Transport Forum, "Shared Mobility Simulations for Auckland," 2017.

[5] International Transport Forum, “Urban Mobility System Upgrade," 2015.

[6] International Transport Forum, “Shared Mobility Simulations for Helsinki," 2017.

[7] S. Ma, Y. Zheng, and O. Wolfson, "Real-Time City-Scale Taxi Ridesharing," IEEE Trans. Knowl. Data Eng., vol. 27, no. 7, pp. $1782-1795$, Jul. 2015.

[8] P. M. Bösch, F. Ciari, and K. W. Axhausen, "Autonomous vehicle fleet sizes required to serve different levels of demand," Transp. Res. Rec., vol. 2542, no. 4, pp. 111-119, 2016.

[9] J. Barceló, Ed., Fundamentals of Traffic Simulation, vol. 145. New York, NY: Springer New York, 2010.

[10] M. P. Linares, L. Montero, E. Lorente, O. Serch, G. Navarro, J. Salmeron, and J. Casanovas, "Analytics tool for assessing innovative mobility concepts, vehicles and city policies (CitScale)," in 2017 5th IEEE International Conference on Models and Technologies for Intelligent Transportation Systems (MTITS), 2017, pp. 385-390.

[11] L. Montero, M. P. Linares, O. Serch, and J. Casanovas-Garcia, "A visualization tool based on traffic simulation for the analysis and evaluation of smart city policies, innovative vehicles and mobility concepts.," in Proceedings of 2017 Winter Simulation Conference, WSC 2017, Las Vegas, NV, USA, December 3-6 ISBN 978-1-5386-3428-8, 2017, pp. 3196-3207.

[12] R Studio Project, “shiny: Web Application Framework for R version 1.3.2 from CRAN.” 2014.

[13] J. Barceló Bugeda, L. Montero Mercadé, and X. Ros Roca, "Virtual mobility lab: a systemic approach to urban mobility challenges," 2018.

[14] Transport Simulation Systems, “Aimsun Microscopic Simulator (v 8.0)." 2014.

[15] J. Salmerón Moya, “Design a BigData Architecture for Simulation Environments (in Spanish)," Universitat Politècnica de Catalunya (UPC) - URI http://hdl.handle.net/2117/91397, 2016

[16] R Development Core Team, "R: A Language and Environment for Statistical Computing," R Foundation for Statistical Computing. 2016.

[17] E. Lorente García, “Data Visualization System for Traffic Simulation results (in Spanish),” Universitat Politècnica de Catalunya (UPC) - URI http://hdl.handle.net pending, 2017.

[18] R. RStudio Project, "Shiny Web Application framework for R." [Online]. Available: https://www.rstudio.com/products/shiny/.

[19] L. Montero, M. P. Linares, J. Casanovas, E. Codina, G. Recio, E. Lorente, and J. Salmerón, "Impact on Network Performance of Probe Vehicle Data Usage: An Experimental Design for Simulation Assessment," J. Adv. Transp., vol. 2018, pp. 1-12, Jun. 2018.

[20] L. Montero Mercadé, M. P. Linares Herreros, J. Casanovas Garcia, G. Recio Domenech, E. Lorente, and J. Salmeron Moya, "Simulation assessment of the new generation navigation strategies," Winter Simul. Conf. 2018 Gothenburg, Sweden december 9-12 Proc., pp. 1-12, 2018.

[21] M. P. Linares, L. Montero, J. Barcelo, and C. Carmona, "A simulation framework for real-time assessment of dynamic ride sharing demand responsive transportation models," in 2016 Winter Simulation Conference (WSC), 2016, pp. 2216-2227.

[22] M. P. Linares, J. Barceló, C. Carmona, and L. Montero, "Analysis and Operational Challenges of Dynamic Ride Sharing Demand Responsive Transportation Models," Transp. Res. Procedia, vol. 21, pp. 110-129, Jan. 2017.

[23] L. Montero, M. Pacheco, J. Barceló, S. Homoceanu, and J. Casanovas, "Case Study on Cooperative Car Data for Estimating Traffic States in an Urban Network," Transp. Res. Rec. J. Transp. Res. Board, vol. 2594, no. 1, pp. 127-137, Jan. 2016.

\section{Author biographies}

MaPaz Linares is a post-doctorate researcher in the field of Mathematical Programming at inLab FIB. She is also an Assistant Professor in the Department of Statistics and Operational research at the Universitat Politècnica de Catalunya. Her research interests include dynamic traffic assignment, traffic simulation and optimization. Contact her at: mari.paz.linares@upc.edu.

Lídia Montero is an associate professor at the Department of Statistics and Operations Research at the Universitat Politècnica de Catalunya. Her research covers simulation-optimization issues, demand modelling, data analysis and models with applications in transport. Contact her at: lidia.montero@upc.edu.

Josep Casanovas is a full professor in Operations Research at the Universitat Politècnica de Catalunya, specializing in simulation systems. He is also the director of inLab FIB (Barcelona informatics school laboratory), an institution that has been very active in transferring technology to business. Contact him at: josepk@fib.upc.edu. 
